\title{
Discovery and profiling of small RNAs responsive to stress conditions in the plant pathogen Pectobacterium atrosepticum
}

\author{
Stanford Kwenda', Vladimir Gorshkov²,3, Aadi Moolam Ramesh'1, Sanushka Naidoo ${ }^{4}$, Enrico Rubagotti ${ }^{5}$,
} Paul R. J. Birch ${ }^{6}$ and Lucy N. Moleleki ${ }^{1^{*}}$

\begin{abstract}
Background: Small RNAs (sRNAs) have emerged as important regulatory molecules and have been studied in several bacteria. However, to date, there have been no whole-transcriptome studies on sRNAs in any of the Soft Rot Enterobacteriaceae (SRE) group of pathogens. Although the main ecological niches for these pathogens are plants, a significant part of their life cycle is undertaken outside their host within adverse soil environment. However, the mechanisms of SRE adaptation to this harsh nutrient-deficient environment are poorly understood.

Results: In the study reported herein, by using strand-specific RNA-seq analysis and in silico sRNA predictions, we describe the sRNA pool of Pectobacterium atrosepticum and reveal numerous sRNA candidates, including those that are induced during starvation-activated stress responses. Consequently, strand-specific RNA-seq enabled detection of 137 sRNAs and sRNA candidates under starvation conditions; 25 of these sRNAs were predicted for this bacterium in silico. Functional annotations were computationally assigned to 68 sRNAs. The expression of sRNAs in P. atrosepticum was compared under growth-promoting and starvation conditions: 68 sRNAs were differentially expressed with 47 sRNAs up-regulated under nutrient-deficient conditions. Conservation analysis using BLAST showed that most of the identified sRNAs are conserved within the SRE. Subsequently, we identified 9 novel sRNAs within the P. atrosepticum genome.

Conclusions: Since many of the identified sRNAs are starvation-induced, the results of our study suggests that sRNAs play key roles in bacterial adaptive response. Finally, this work provides a basis for future experimental characterization and validation of sRNAs in plant pathogens.
\end{abstract}

Keywords: Small RNAs, Strand-specific RNA-seq, Pectobacterium atrosepticum, in silico prediction, Transcriptome, Riboswitches, 5' UTR, 3' UTR

\section{Background}

The importance of small RNAs (sRNAs) in bacterial gene expression regulation is now broadly appreciated $[1,2]$. sRNAs play essential regulatory roles in diverse processes including metabolic reactions, stress response, biofilm formation and pathogenesis [3]. They act as either activators or repressors of proteins and mRNAs. The length of most of the bacterial sRNAs ranges between 50 and 300 but can reach up to 500 nucleotides

\footnotetext{
* Correspondence: lucy.moleleki@up.ac.za

'Department of Microbiology and Plant Pathology, Forestry and Agricultural Biotechnology Institute (FABI), University of Pretoria, Pretoria, South Africa

Full list of author information is available at the end of the article
}

[4]. The best studied bacterial regulatory sRNAs are those that act through base-pairing interactions with target RNAs, usually modulating gene expression posttranscriptionally by controlling the translation and stability of mRNAs. The majority of these are trans-acting sRNAs found within intergenic regions (IGRs). Trans-acting sRNAs typically regulate mRNAs encoded at different genomic locations on the chromosome in response to changes in environmental conditions [1]. Furthermore, trans-encoded sRNAs tend to have limited complementarity with their target RNAs and require the RNA chaperone Hfq to facilitate their pairing with mRNA targets [4]. In contrast, cis-encoded antisense RNAs (asRNAs), also 
referred to as naturally occurring RNAs, are expressed on reverse strands opposite to annotated genes and have extensive complementarity with their target mRNAs [4]. Antisense RNAs are thought to play physiological roles such as repression of genes encoding potentially toxic proteins [5]. Additional roles of asRNAs include blocking the translation of mRNA transcripts encoded on the opposite strand and directing their RNAse III-mediated cleavage [4]. Other important classes of sRNAs include 1) riboswitches (leader sequences), which form part of the mRNA they regulate and usually present in the $5^{\prime}$ UTR regions; 2) sRNAs which interact with proteins and modify their activities by mimicking their RNA or DNA targets, and 3) sRNAs with intrinsic regulatory activities [4].

The advent of RNA-seq for the resolution of messenger and structural RNAs has facilitated the analysis of vast numbers of sRNAs with increased sensitivity [6, 7]. An additional benefit of RNA-seq approaches is that information about the direction of transcription can be resolved using directional RNA-seq (strand-specific RNA-seq; ssRNA-seq). This information is important for the detection of non-coding (nc) RNAs as well as $5^{\prime}$ and $3^{\prime}$ untranslated regions (UTRs), antisense transcripts and determination of overlapping features within the genome [6]. Combining deep sequencing with computational (in silico) prediction methods is emerging as an important approach for sRNA detection in bacterial genome sequences $[8,9]$.

Pectobacterium atrosepticum is an important plant pathogen belonging to the bacterial family Enterobacteriaceae [10]. This pathogen causes major yield losses globally through blackleg disease on potato plants in the field and potato tuber soft rot diseases during post-harvest storage. Most of the information on pectobacteria concerns their interaction with plant hosts, and little is known about how these bacteria spend much of their life outside of the host [11]. However, it is known that P. atrosepticum is able to utilize various adaptive programs that enable bacteria to survive under adverse conditions [12, 13]. In a previous study, we showed that realization of these programs under nutrient-deficient conditions (starvation) is coupled with an increased transcript abundance of stress responsive genes in P. atrosepticum, and bacterial cells undergo morphological and ultrastructural changes [14]. In the current study we have evaluated the possible participation of sRNAs in bacterial starvation-induced stress response.

Few experimental studies on sRNAs have been carried out in $P$. atrosepticum. A well-known regulatory sRNA in $P$. atrosepticum is $r s m B$. This sRNA binds the RsmA protein, which is a homologue of Escherichia coli CrsA, a carbon storage regulator, and modulates its activity. In P. atrosepticum the $\mathrm{RsmA} / \mathrm{rsmB}$ system regulates the production of virulence factors [15-17]. Moreover, a regulatory RNA antisense to the $\operatorname{expI}$ gene transcript, which encodes the synthase of mediators of quorum sensing (acyl-homoserine lactones), was found recently in P. atrosepticum [18].

In the present study, identification of sRNAs in the complete genome of $P$. atrosepticum SCRI1043 was undertaken using in silico prediction and experimental validation via strand-specific RNA-sequencing. Both true (and/or known) and potentially novel sRNA candidates expressed under starvation conditions were identified. Differential expression analysis indicated that many of these sRNAs increase in abundance during exposure of bacteria to starvation compared to rich medium conditions, suggesting an important role of sRNAs in the survival of $P$. atrosepticum cells during nutrient deficiency induced stress.

\section{Results and discussion}

\section{Strand-specific RNA-seq detection of $P$. atrosepticum} sRNAs under starvation-conditions

For experimental detection of sRNAs in P. atrosepticum SCRI1043, we used a combination of in silico and directional whole-transcriptome cDNA sequencing (strandspecific RNA-seq) (Fig. 1). The experimental approach for determination of sRNA in P. atrosepticum is outlined in Fig. 1a. A total of 27.4 and 26.1 million paired-end (PE) reads were obtained from nutrient rich and starvation conditions, respectively. By using SAMtools [19], PE reads mapped to each strand were extracted. Thus, enabling visualization of the sequence (PE) read alignments on the genome in a strand-specific manner. Visual inspections enabled the identification of candidate sRNA transcripts by manually analysing the position of $\mathrm{PE}$ reads with respect to annotated protein-coding regions (CDS). This can be a particularly powerful approach to identify sRNAs and resolve their genomic positions because reads that map to intergenic regions may represent previously unannotated transcriptionally active non-coding sRNAs [20]. Only sRNA candidates with a length between 50 to 500 nucleotides were considered to be true positive sRNAs candidates. This technique enabled identification of a total of 137 sRNA candidates expressed under starvation condition (Additional file 1: Table S1). These candidate sRNAs were classified into four distinct sRNA groups based on their position in relation to adjacent CDSs: IGR/ transencoded sRNAs, asRNA, 5' UTR (riboswitches), and 3' UTR sRNAs (Fig. 2). An in silico approach (described in the section below) was employed to determine the putative transcriptional start sites (TSS) of the identified 137 sRNAs and to resolve their $5^{\prime}$ ends. Only predicted TSS with transcription factor binding sites were considered as bona fide promoters. Thus, using this filter, TSS were identified upstream of 118 sRNA genes (Additional file 2: Table S2). 
a

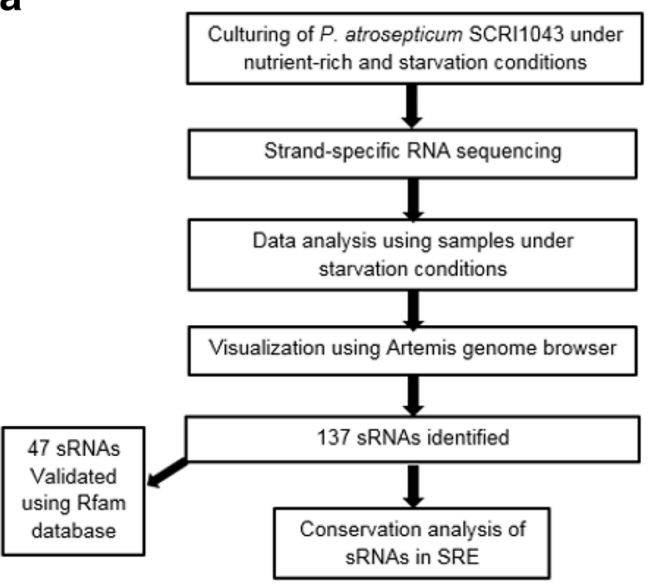

C b

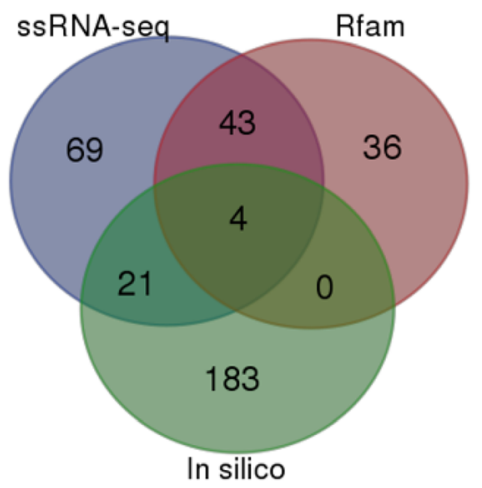

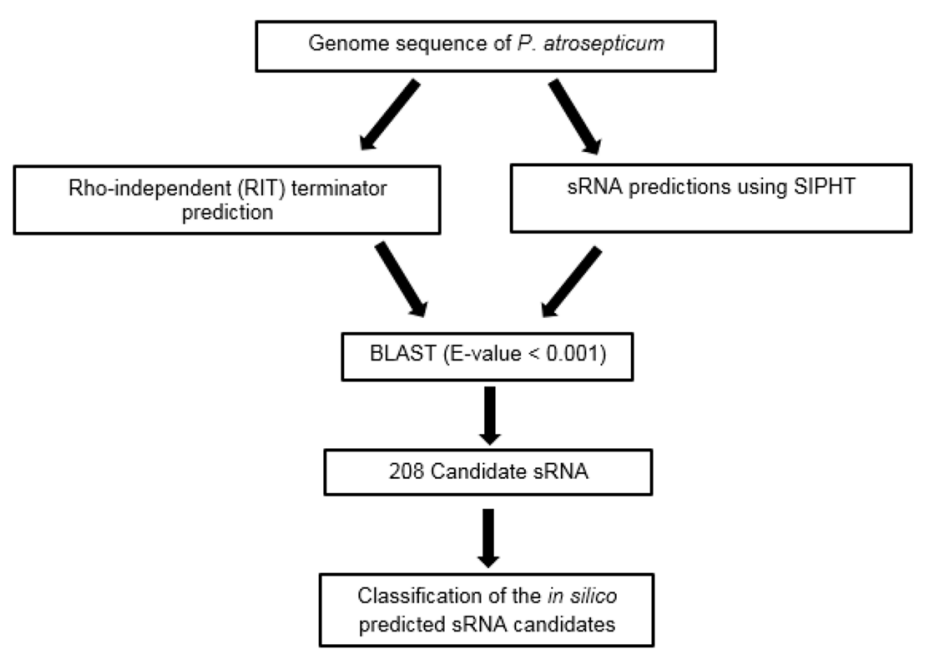

Fig. 1 Scheme for sRNA identification. a Determination of sRNA using strand-specific RNA seq of $P$. atrosepticum cultured under starvation conditions. b Comparison of sRNAs identified by strand-specific RNA-seq with sRNA candidates predicted for $P$. atrosepticum in Rfam database and sRNAs predicted computationally in this study. c Computational (in silico) sRNA prediction

\section{Identification of 3' UTR encoded sRNAs}

We identified 15 sRNAs encoded within the 3' UTR regions of mRNA (referred to in this study as $3^{\prime}$ UTR sRNAs) (Fig. 2). It is now appreciated that sRNAs not only originate from intergenic regions as independent transcripts but are also transcribed from 3' regions of coding mRNA [21]. These 3' UTR sRNAs are generated either by means of mRNA transcript processing or as primary transcripts from an internal promoter within the mRNA coding sequence as in the case of dapZ sRNA [22]. Thus, based on how they are produced, 3' UTR encoded sRNAs can be divided into 2 groups, that are: 1) sRNAs transcribed from an independent promoter located inside the overlapping mRNA gene or 3' UTR region (Type 1); and 2) sRNAs which are originated from the processing of the parent mRNA (Type 2) [23]. Hence we used our ssRNA-seq data to determine whether the identified 3' UTR embedded sRNAs are transcribed independently from the parent mRNA. Ten 3' UTR sRNAs were considered to be independently transcribed based on comparisons of sRNA and parent mRNA RPKM (reads per kilobase of transcript per million mapped reads) values and the presence or absence of an internal promoter (Table 1). To determine the putative $5^{\prime}$ ends and fundamental types of the detected $3^{\prime}$ UTR sRNAs based on their biogenesis, we extracted each sRNA sequence plus $200 \mathrm{nt}$ upstream of the start position of each sRNA and performed promoter predictions using BPROM program (http://www.softberry.com/berry. phtml?topic $=$ bprom\&group=programs\&subgroup $=$ gfindb). This approach led to the identification of 14 distinct putative promoter sites (transcriptional start sites; TSS) embedded within the coding or 3' UTR regions of the parent mRNA upstream of each 3' UTR sRNA gene (Table 1). In 


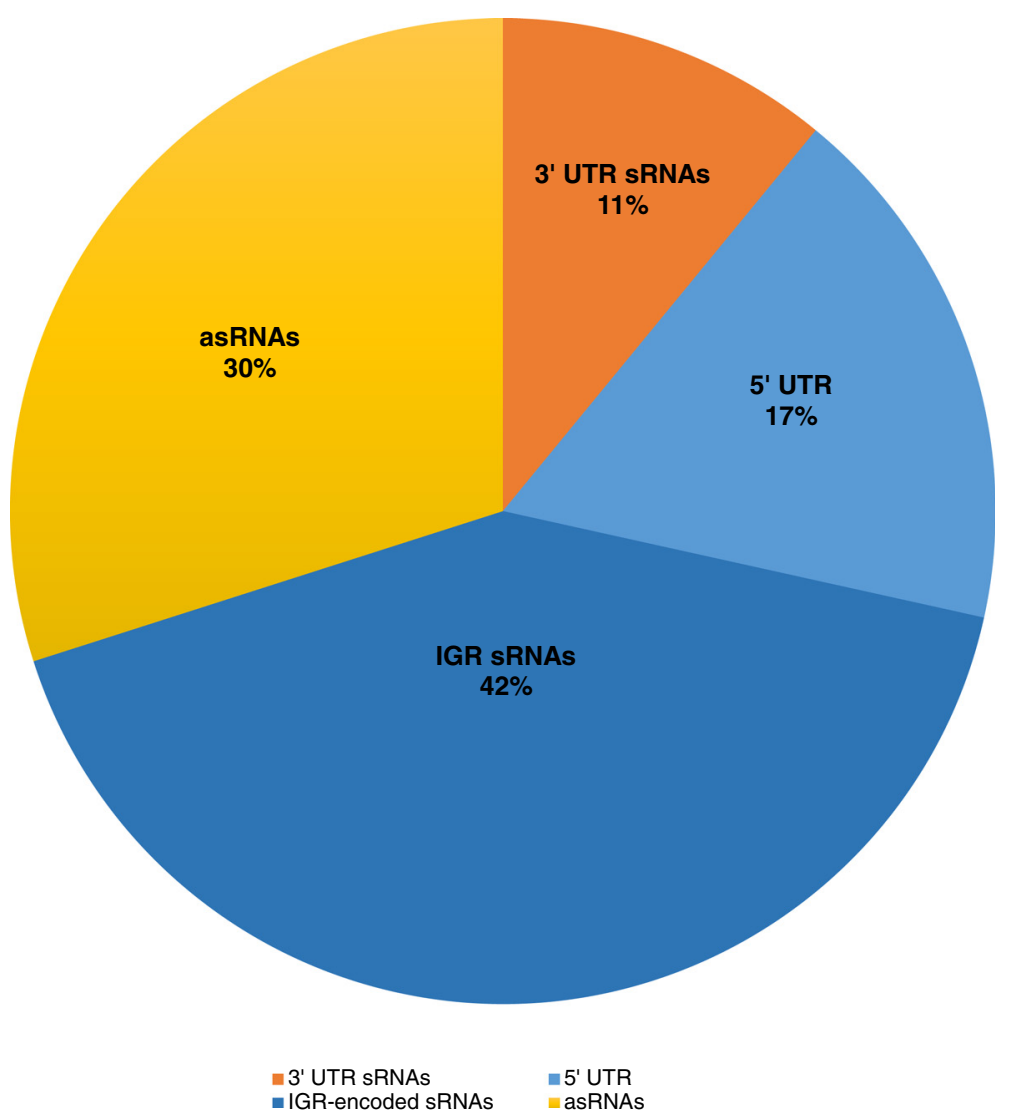

Fig. 2 Classification of sRNAs identified using ssRNA-seq into five classes: These include; IGR/ trans-encoded sRNAs, asRNA, 5'UTR (riboswitches), 3' UTR and sense sRNAs (seRNAs), based on their proximity and location with regards to CDS regions

addition, transcription factor binding sites were also detected within the predicted promoter regions. Taken together, the presence of putative internal promoter sites upstream of sRNAs TSS and the predicted transcriptional factor binding sites for each promoter, strongly suggests that fourteen 3' UTR sRNAs are type 1. Nine of which were also differentially expressed compared to their parent mRNAs based on RPKM values, further indicating evidence of independent expression. The remaining sRNA reg_seq13 could be a product of mRNA processing, thus type 2 since no internal promoters supported by transcription binding sites were predicted for this sRNA. Overall, since the sRNA $5^{\prime}$ ends and subsequent TSSs were predicted computationally, we were not able to determine whether these sRNAs possessed the characteristic $5^{\prime}$-triphosphate $\left(5^{\prime}\right.$-PPP) cap common to type 1 sRNAs in this present study.

The 137 sRNAs identified using strand-specific RNAseq approach were checked against known $P$. atrosepticum SCRI1043 non-coding RNA descriptions on the Rfam database [24]. For this analysis, all descriptions for tRNAs, rRNAs and CRISPR RNAs were excluded. This also served to assess the efficiency of the strand-specific RNA- seq method in detecting sRNA transcripts. In total $56.6 \%$ (47/83) of the known P. atrosepticum sRNAs in the Rfam database were identified using ssRNA-seq of cells cultured under starvation conditions (Fig. $1 \mathrm{~b}$ and Additional file 1: Table S1).

\section{Computational prediction of sRNA in the Pectobacterium atrosepticum genome}

Even though ssRNA-seq is a powerful tool for identification of sRNAs, it might be subject to some limitations. For example, since the formation of particular sRNAs is highly dependent on culture conditions, it is not possible to unravel the whole pool of sRNAs that is encoded in the genome of the target microorganism within the frameworks of a given experiment. Consequently, a combination of experimental and computational identification of sRNA is often seen as a more comprehensive approach towards identification of sRNAs $[25,26]$. Hence, in addition to ssRNA-seq, an in silico sRNA analysis was performed according to computational methods implemented previously [27], with some modifications (see Fig. 1c for a schematic representation of the computational prediction strategy). 
Table 1 3' UTR encoded sRNAs

\begin{tabular}{|c|c|c|c|c|c|c|c|c|c|c|c|c|}
\hline & \multicolumn{4}{|c|}{ sRNA } & \multicolumn{2}{|l|}{ RPKMs } & \multirow{2}{*}{$\begin{array}{l}\text { Expression } \\
\text { (based on RPKMs) }\end{array}$} & \multirow{2}{*}{$\begin{array}{l}\text { Predicted } \\
\text { promoters }\end{array}$} & \multicolumn{3}{|c|}{ sRNA promoter and start site } & \multirow[t]{2}{*}{ Transcription Factor } \\
\hline sRNA name & Parent mRNA & Start & end & length & sRNA & mRNA & & & -35 & -10 & TSS & \\
\hline fwd_4 & $r b s B$ & 14355 & 14537 & 183 & 596.8 & 102.8 & Independent & 1 & 14317 & 14342 & 14357 & rpoD17, cynR, rpoD15, rpoD16, phoB \\
\hline fwd_6 & polA & 28634 & 28755 & 122 & 549.8 & 110.5 & Independent & 1 & 28656 & 28676 & 28691 & cytR, arcA, crp, rpoD15, rpoD17 \\
\hline fwd_15 & ECA0044 & 55039 & 55291 & 253 & 66.8 & 27.4 & Independent & 1 & 55009 & 55029 & 55044 & rpoD17, fis, rpoD15, rpoD16, phoB \\
\hline fwd_19 & $\exp 1$ & 126355 & 126501 & 147 & 338.5 & 588.1 & Co-expression & 1 & 126418 & 126435 & 126450 & metR, rpoD16 \\
\hline reg_seq13 & aldA & 139913 & 140154 & 242 & 136.8 & 30.4 & Independent & 1 & 140046 & 140063 & 140078 & \\
\hline reg_seq27 & ECA0332 & 380584 & 380826 & 243 & 1660.5 & 389.3 & Independent & 1 & 380610 & 380631 & 380646 & metJ \\
\hline reg_seq31 & ECA0449 & 515673 & 515910 & 238 & 363.3 & 528.7 & Co-expression & 1 & 515790 & 515813 & 515828 & glpR, inf, argR2, nagC, argR2, fnr, fis \\
\hline \multirow[t]{2}{*}{ reg_seq43 } & $m d H$ & 758603 & 758857 & 255 & 1759.1 & 1895.7 & Co-expression & 2 & 758751 & 758770 & 758789 & glpR, fis, arcA, purR \\
\hline & & & & & & & & & 758444 & 758464 & 758479 & \\
\hline reg_seq142 & ECA2516 & 2832294 & 2832530 & 237 & 514.2 & 77.5 & Independent & 1 & 2832185 & 2832205 & 2832220 & purr, rpoD16 \\
\hline fwd_rfam4 & rpil & 255260 & 255321 & 62 & 775.5 & 1856.1 & Independent & & & & & Irp, hns \\
\hline comp_seq5 & $g \ln A$ & 34992 & 35234 & 243 & 858.8 & 1209.3 & Co-expression & 1 & 35262 & 35241 & 35226 & rpoD15, rpoD16, phoB \\
\hline comp_seq11 & $\operatorname{simA}$ & 164026 & 164334 & 309 & 196.4 & 454.0 & Independent & 1 & 164395 & 164375 & 164360 & arcA, rpoD17, rpoD15, rpoD16, phoB \\
\hline \multirow[t]{2}{*}{ comp_seq130 } & ECA2950 & 3295111 & 3295347 & 237 & 197.9 & 222.9 & Co-expression & 2 & 3295196 & 3295176 & 3295161 & rpoD16, argR, arcA, inf \\
\hline & & & & & & & & & 3295506 & 3295485 & 3295470 & \\
\hline rev_rfam 22 & $g / p C$ & 4651380 & 4651485 & 109 & 348.2 & 40.8 & Independent & 1 & 4651583 & 4651562 & 4651547 & Ada, rpoE, tyrR, fur, fur \\
\hline dapZ & ECA3872 (dapB) & 4332178 & 4332288 & 111 & 20.9 & 110.8 & Independent & 1 & 4332302 & 4332284 & 4332269 & Ihf, $\operatorname{argR2}$, rpoD16, $\operatorname{argR}$, fis, crp \\
\hline
\end{tabular}


An initial step towards in silico sRNA candidate disclosure consisted of identification of predicted rhoindependent terminators (RITs) in the P. atrosepticum SCRI1043 genome. Since about $72 \%$ of known sRNAs located within IGRs possess a RIT, computational methods based on prediction of RIT signature sequences have emerged as valuable algorithms for the detection of sRNA molecules [8, 27]. In intergenic and antisense to annotated open reading frames (ORF) in the $P$. atrosepticum SCRI1043 genome we detected a total of 1598 putative terminators (including both canonical and non-canonical terminators' candidates) with the 'Greatest $\Delta G$ ' i.e. the most negative $\Delta G$ (free Gibbs energy) value. From the 1598 putative sRNA identified, 1165 were filtered out and excluded from further analysis due to the fact that their RITs were located less than 60 nucleotides downstream from stop codons of preceding annotated ORFs within the same strand. This resulted in identification of 433 sRNA candidates of 226-248 nt in length (Additional file 3: Table S3). To be more confident about the accuracy of the rho-independent terminator based prediction strategy used, a second prediction tool (SIPHT) [28] was employed. Herewith, the filtered set of sRNA candidate signatures was compared against sRNA predictions for $P$. atrosepticum SCRI1043 from the SIPHT web interface by means of BLAST local pairwise alignments using the genomic similarity search tool YASS [29], with standard parameters. Each comparison was made on both regular and complementary strands separately. As a result, a total of 105 and 101 matches (E-value $<0.001$ ) were identified, partially or fully overlapping, for the forward and complementary strands, respectively. This additional filtering step combining comparative genomics with RIT based predictions yielded 206 sRNA candidates in P. atrosepticum SCRI1043 (Additional file 4: Table S4). Similarly to sRNA detected using ssRNA-Seq, predicted sRNAs were further classified into five distinct sRNA groups based on their position in relation to adjacent CDSs (results not included).

\section{Comparison of RNA-seq results with computational sRNA predictions}

The 208 candidate sRNAs identified computationally were compared to the 137 sRNA transcripts identified using ssRNA-seq. Only 25 of the in silico predicted sRNA candidates were also identified by RNA sequencing (Table 2). Such an incomplete overlap between computational sRNA predictions and deep sequencing detection has been noted in previous studies $[2,8,9,30]$. It is possible that the discrepancy observed here could be largely because experimental detection of sRNAs was restricted to sRNAs expressed under one condition, viz starvation. Hence, it may well be that increasing the number of conditions in which RNA is harvested could lead to bridging the gap between in silico predicted and ssRNAseq identified sRNAs. Lastly, the disparity could be due to the presence of false positive in silico predictions as well as the elimination of sRNAs associated with RITs in close proximity to CDS regions when using RIT identification based in silico predictions. Nonetheless, the lengths of the majority of the in silico predicted sRNA transcripts were comparable to the sizes deduced from the strand-specific RNA-seq sRNA detections for the confirmed sRNA candidates.

\section{Functional annotation of RNA-seq detected sRNAs}

To describe and assign biological functions to the 137 sRNAs detected by strand-specific RNA-seq (including those confirmed by in silico predictions), we used the Rfam database (version 11.0) [31] and the RNAspace platform [32]. The RNAspace platform comprises a suite of ncRNA prediction tools. Similarity searches on the RNAspace platform were restricted to comparative analysis and homology searches using BLAST/ YASS (sequence homology tools) against the Rfam 10.0 seed database and three RNA motif search tools, DARN, ERPIN and INFERNAL. In total, 68 sRNAs representing true (and/or known), previously described sRNA sequences were assigned into 6 functional classes (E-value $<0.001$ ), and these included: 1 ribozyme, 21 riboswitches (consisting of 6 types), 14 RNA elements (10 different types), 30 sRNAs (including 9 Hfqbinding sRNAs), 1 asRNA and 1 tmRNA (Table 3). Amongst these, we characterized 13 sRNA sequences which were previously uncharacterized within the $P$. atrosepticum genome by means of Blast $(\mathrm{e}$-value $<0.001)$ and secondary structure predictions using the RNAfold Webserver [33]. No functional classes were assigned to the remaining 69 sRNAs computationally, suggesting that they could be potentially novel sRNA candidates in $P$. atrosepticum.

Most of the detected riboswitches in this study corresponded to thiamine pyrophosphate (TPP) (Thi-box) riboswitches (Table 3). Bacterial riboswitches are embedded within the leader sequences (5' UTR regions) of numerous metabolic genes and act by repressing or activating their cognate genes at the translational level in gram-negative bacteria [34]. Most thiamin-regulated genes encode transporters in different bacterial organisms [35]. For example, TPP riboswitches identified are present upstream of genes involved in potassium transport $(\operatorname{trk} D)$, amino acid biosynthesis $(\arg G)$, and genes related to the biosynthesis of secondary metabolites $(m e n D)$. Generally, TPP riboswitches are found upstream (5' UTR regions) of many genes key in metabolic processes which use TPP as a cofactor [35]. In this study, we also detected other riboswitches other than TPP-type riboswitches, that include Flavin mononucleotide (FMN), glycine, lysine, yybP-ykoy and MOCO RNA motif riboswitches. 
Table 2 In silico predicted sRNA candidates confirmed by strand-specific RNA-seq

\begin{tabular}{|c|c|c|c|c|c|}
\hline sRNA candidate & sRNA class & sRNA type & sRNA start & sRNA end & sRNA length \\
\hline reg_seq3 & 3'UTR: polA & & 28634 & 28755 & 122 \\
\hline reg_seq3b & IGR & spot42 sRNA & 28756 & 28882 & 127 \\
\hline reg_seq13 & 3' UTR: aldA & & 139913 & 140154 & 242 \\
\hline reg_seq27 & 3' UTR: ECA0332 & TPP riboswitch & 380584 & 380826 & 243 \\
\hline reg_seq27b & 5' UTR: icc & isrH (Hfq binding sRNA) & 380825 & 380917 & 93 \\
\hline reg_seq31 & 3' UTR: ECA0449 & & 515673 & 515910 & 238 \\
\hline reg_seq34 & 3' UTR: topB & STAXI sRNA & 601635 & 601862 & 228 \\
\hline reg_seq34b & antisense: ECA0527 & STAXI sRNA & 601860 & 602087 & 228 \\
\hline reg_seq43 & 3' UTR: mdh & Glycine riboswitch & 758603 & 758857 & 255 \\
\hline reg_seq67 & IGR & & 1185902 & 1186147 & 246 \\
\hline reg_seq70 & antisense: ECA1096 & & 1225748 & 1226182 & 435 \\
\hline reg_seq76 & 5' UTR: mend & TPP riboswitch & 1379050 & 1379349 & 300 \\
\hline reg_seq109 & antisense: osmB & $\mathrm{TPP} / \mathrm{isrH}$ & 2217586 & 2217946 & 361 \\
\hline reg_seq129 & IGR & Trp leader & 2602697 & 2602931 & 235 \\
\hline reg_seq133 & 5' UTR: ansA & RtT and TPP & 2651356 & 2651598 & 243 \\
\hline reg_seq142 & 3' UTR: ECA2516 & & 2832294 & 2832530 & 237 \\
\hline comp_seq5 & 3' UTR: glnL & TPP/ isrH & 34992 & 35234 & 243 \\
\hline comp_seq11 & 3' UTR: s/mA & isrH & 164026 & 164334 & 309 \\
\hline comp_seq16 & 5'UTR: ECA0353 & TPP & 403257 & 403655 & 399 \\
\hline comp_seq49 & IGR & TPP & 1218529 & 1218729 & 201 \\
\hline comp_seq55 & 5' UTR: ECA1196 & $\mathrm{TPP} / \mathrm{isr} \mathrm{H}$ & 1358123 & 1358268 & 146 \\
\hline comp_seq111 & IGR & TPP/ isrH & 2881355 & 2881546 & 192 \\
\hline comp_seq130 & 3' UTR: ECA2950 & TPP and RtT & 3295111 & 3295347 & 237 \\
\hline comp_seq204 & 5' UTR: $\operatorname{sot} B$ & & 4828158 & 4828349 & 192 \\
\hline comp_seq217 & 5' UTR: ECA4506 & & 5046219 & 5046427 & 209 \\
\hline
\end{tabular}

Some of the detected RNA elements (leader sequences) were located upstream of operons or genes involved in biosynthesis of amino acids including leucine, histidine and tryptophan biosynthesis; and polysaccharide synthesis (Additional file 1: Table S1). It therefore seems plausible that most of the detected cis-regulatory elements are engaged in regulating processes involving substrate transport and biosynthesis in $P$. atrosepticum.

\section{Conservation analysis of predicted sRNAs}

The vast majority of known sRNAs are typically highly conserved across genera [36]. We therefore analysed the conservation of identified sRNAs in $P$. atrosepticum SCRI1043 in five soft rot Enterobacteriaceae species whose complete genome sequences are available on GenBank. The 68 true/ known sRNA sequences with assigned functional classes were used for the conservation analysis. BLASTn analysis $(\mathrm{E}$-value $<0.0001)$ using the YASS tool against $P$. carotovorum subsp. carotovorum PC1, $P$. wasabiae WPP163, Pectobacterium spp. SCC3193, Dickeya dadantii Ech703 and D. zeae Ech1591 complete genome sequences revealed that most sRNAs are conserved within the soft rot bacterial species with 42 sRNAs (including 13 trans-encoded sRNAs, 18 riboswitches, 10 RNA elements, and 1 asRNAs) being present in all five SRE species (Fig. 3 and Additional file 5: Table S5). The high conservation of sRNAs within the SRE species emphasizes their regulatory importance in these bacteria. Six IGR sRNAs were conserved only in the Pectobacterium genus and belonged to two RNA families; namely $s t y R-44$, and $\operatorname{crcB}$ RNA motif (fluoride riboswitch) sensing fluoride ions and regulating the $\operatorname{crcB}$ gene (hypothetical protein) which possibly encodes a protein that functions by removing excess fluoride ions from the cell.

To be more confident with the 69 potentially novel sRNA candidates detected by ssRNA-seq, we filtered and screened them by checking their conservation within the five representative SRE strains using sequence similarity analysis. Nine of these candidate sRNAs had high sequence conservation (100\% identity and coverage) within SRE strains and only single hits from the BLAST analysis and therefore were considered as novel sRNAs (Table 4). 
Table 3 Functional annotation of the 68 true (and/or known) sRNAs identified by strand-specific RNA-seq

\begin{tabular}{|c|c|c|c|c|}
\hline sRNA name & sRNA type & Characterized in this study (RNAspace) & Previously characterized (Rfam) & Total \\
\hline RNaseP & Ribozyme & & 1 & 1 \\
\hline tmRNA & & & 1 & 1 \\
\hline \multicolumn{5}{|l|}{ Cis-regulators } \\
\hline & Riboswitches & & & \\
\hline TPP riboswitch & & 6 & 3 & 9 \\
\hline TPP or isrH & & 8 & & 8 \\
\hline glycine riboswitch & & 2 & 2 & 4 \\
\hline FMN & & & 1 & 1 \\
\hline lysine riboswitch & & 1 & & 1 \\
\hline MOCO_RNA_motif & & & 1 & 1 \\
\hline \multirow[t]{2}{*}{ yybP-ykoY } & & & 1 & 1 \\
\hline & RNA elements & & & \\
\hline alpha_RBS & & & 1 & 1 \\
\hline $\operatorname{cspA}$ & & & 2 & 2 \\
\hline greA & & & 1 & 1 \\
\hline his_leader & & & 1 & 1 \\
\hline JUMPStart & & & 2 & 2 \\
\hline leucine operon leader & & & 1 & 1 \\
\hline P26 & & & 1 & 1 \\
\hline rne5 & & & 1 & 1 \\
\hline RtT & & 1 & 2 & 3 \\
\hline trp_leader & & & 1 & 1 \\
\hline
\end{tabular}

trans-encoded sRNA

SRNA

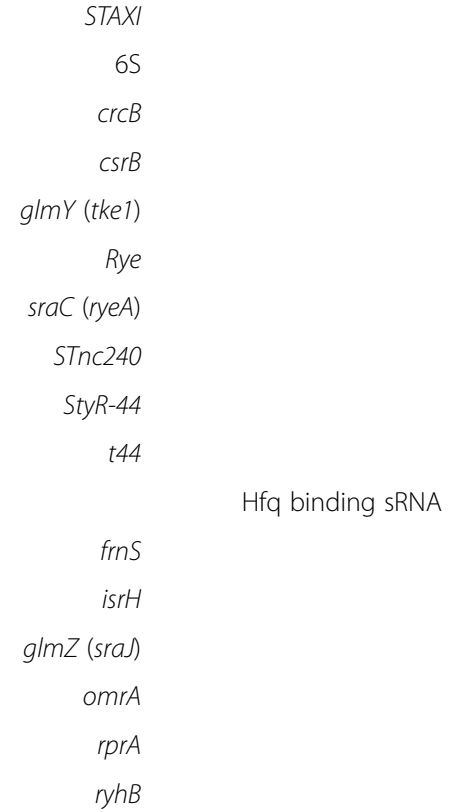


Table 3 Functional annotation of the 68 true (and/or known) sRNAs identified by strand-specific RNA-seq (Continued)

\begin{tabular}{|c|c|c|c|}
\hline ryeB (sdsR) & & 1 & 1 \\
\hline sgrs & & 1 & 1 \\
\hline spot 42 & & 1 & 1 \\
\hline antisenseRNA (asRNA) & asRNA & & \\
\hline $\mathrm{HPnC0260}$ & & 1 & 1 \\
\hline Total & 21 & 47 & 68 \\
\hline
\end{tabular}

To validate the expression and lengths of the nine novel sRNAs, reverse transcription PCR (RT-PCR) was performed on CDNA of bacteria cells cultured under starvation conditions (Fig. 4). For each of the cDNA samples, a single amplicon that corresponded to the sRNA transcript size identified by ssRNA-seq was observed. As an additional validation step, the nucleotide bases of observed amplicons were confirmed by sequencing and alignment to respective sRNA sequences (Additional file 6: Table S6).

\section{Differential expression of sRNAs under nutrient-rich and starvation conditions}

Application of strand-specific RNA-seq to study the transcriptome of $P$. atrosepticum uncovered an abundance of sRNAs including antisense transcripts, intergenic sRNAs and cis-encoded regulatory elements. The number of RNA-seq reads mapping to individual sRNA sequences provides a realistic assessment of relative transcript abundance [37], thus enabling quantification of differential expression of the sRNA transcripts in $P$. atrosepticum cells existing under nutrient-rich and nutrient-deficient (starvation) conditions. The differential expression of sRNAs when growth conditions are changed could suggest potential functions and clarify conditions that induce or repress formation of specific
sRNAs [38]. Hence, in order to understand the expression profiles of sRNAs in response to carbon and phosphorus starvation, we compared expression patterns of $P$. atrosepticum cells under nutrient-rich and nutrientdeficient (starvation) conditions. Based on the combined statistics of edgeR package [39] (dispersion $=0.04$; qvalue $<0.1$ ), and Gfold algorithm (v.1.1.4) [40], which uses a posterior distribution of log fold change for determining expression changes in experiments with single biological replication, thus, overcoming the shortcomings of relying on statistics based on p-value when biological replication is lacking [40]. Subsequently, only sRNAs with significant differential expression from edgeR and Gfold analyses were considered. Thus, a total of 68 sRNA candidates were differentially expressed (Additional file 7: Table S7). Of these, 47 sRNAs were up-regulated under nutrient-deficient conditions (Additional file 7: Table S7) suggesting that they are likely involved in regulatory mechanisms of stress response or adaptation in $P$. atrosepticum. To validate expression profiles identified by ssRNA-seq, we performed reverse transcription quantitative PCR (RTqPCR) using three biological replicates, on eight selected sRNAs that were differentially expressed under nutrient-rich and starvation conditions. The RT-qPCR

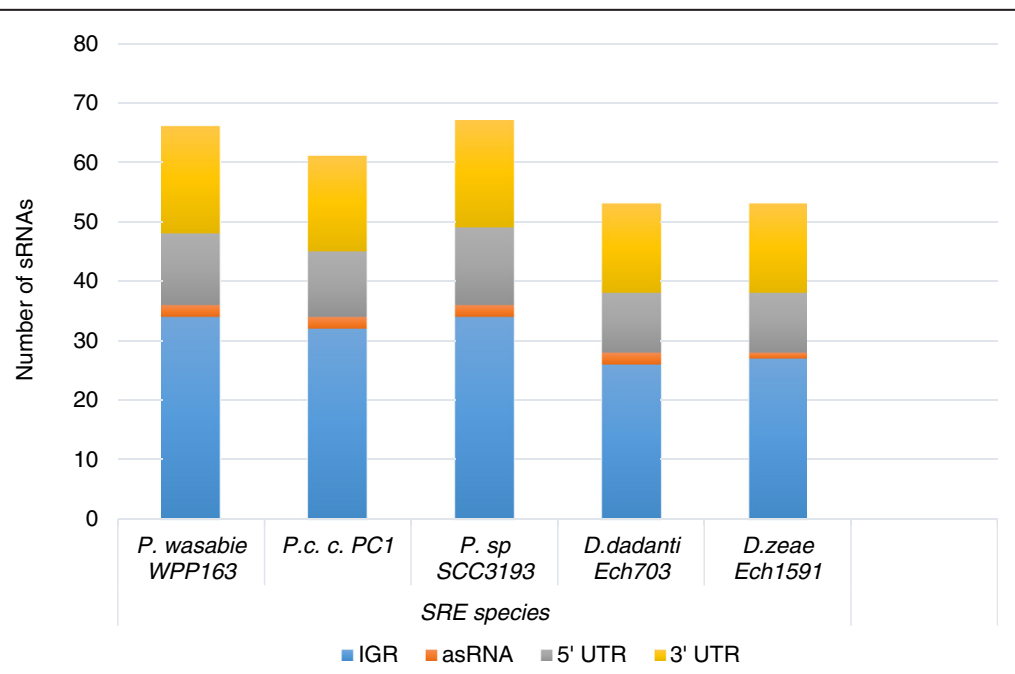

Fig. 3 Summary of sRNAs conserved in soft rot Enterobacterieace 
Table 4 Novel sRNA candidates obtained using conservation analysis

\begin{tabular}{cccr}
\hline sRNA Name & Strand & Length & \multicolumn{1}{c}{ sRNA Class } \\
\hline rev_11 & - & 420 & asRNA: ECA0328 \\
rev_13 & - & 354 & asRNA: ECA0388 \\
rev_24 & - & 489 & asRNA: rCsC \\
rev_39 & - & 300 & 5' UTR: ilvG \\
rev_41 & - & 480 & IGR/ 5' UTR: bcsB \\
fwd_6 & + & 122 & 3' UTR: ECA3097 \\
fwd_42 & + & 480 & 5' UTR: zipA \\
fwd_44 & + & 336 & asRNA: ECA0910 \\
fwd_72 & + & 426 & asRNA: gudP \\
\hline
\end{tabular}

results confirmed expression patterns of these eight sRNA transcripts and validated our RNA-seq data (Fig. 5). Selected examples are discussed below.

We noticed that rprA was up-regulated ( 1.5-fold) in $P$. atrosepticum under carbon-starvation conditions (Fig. 5 and Additional file 7: Table S7). The sRNA rprA acts by increasing (positively regulating) the translation of rpoS gene transcript $[41,42]$. RpoS is a sigma factor that controls the expression of stress responsive genes in bacteria during adverse conditions and stationary phase. We observed that the expression of rpoS gene in $P$. atrosepticum was higher during starvation than under nutrient-rich conditions (data not shown). This observation is consistent with previous data demonstrating that RpoS is a principal regulator of the general stress response in bacteria allowing cells to survive environmental challenges as well as prepare for subsequent stresses [43]. This is also consistent with our previous observations, demonstrating that rpoS gene expression increases significantly in $P$. atrosepticum under stress conditions [14]. Generally, the regulation of rpoS gene expression is known to be modulated at the translational level by at least four sRNA, namely, $\operatorname{arc} Z, d s r A$, $\operatorname{rpr} A$, and oxyS in response to temperature, osmotic shock, oxidative stress and nutrient deprivation in $E$. coli $[41,44]$. Hence, increased rprA expression observed in our study in $P$. atrosepticum under nutrient starvation conditions is likely to promote the enhanced translation of rpoS mRNA during adaptation of bacteria to starvation conditions.

ryhB2, a 106 nucleotide paralogue of $r y h B$ sRNA, was up-regulated by a 15 -fold magnitude in $P$. atrosepticum under nutrient-starvation conditions (Fig. 5). Generally, $r y h B$, regulates iron metabolism, including its acquisition and assimilation. $r y h B$ acts by down-regulating expression of genes encoding iron-storage and iron-using proteins when iron is in limited supply. The main target genes for $r y h B$ include the $s d h C D A B$ operon encoding succinate dehydrogenase and $\operatorname{sod} B$ which encodes the iron-dependent superoxide dismutase [45]. ryhB expression level is usually inversely correlated with expression levels of the mRNA for the $s d h C D A B$ operon [46]. This is consistent with our observations for $P$. atrosepticum: the transcription of the $s d h C D A B$ operon was reduced under starvation conditions compared to the growthpromoting ones (Fig. 6).

Starvation conditions also induced the expression of $\operatorname{glm} Z$ ( 2-fold increase) and $g \operatorname{lm} Y$ (5-fold increase) sRNAs in $P$. atrosepticum (Fig. 5). In enteric bacteria, these two sRNAs regulate amino sugar metabolism by activating the expression of glmUS operon which encodes the glucosamine-6-phosphate synthase, an essential enzyme in amino sugar metabolism [47]. The

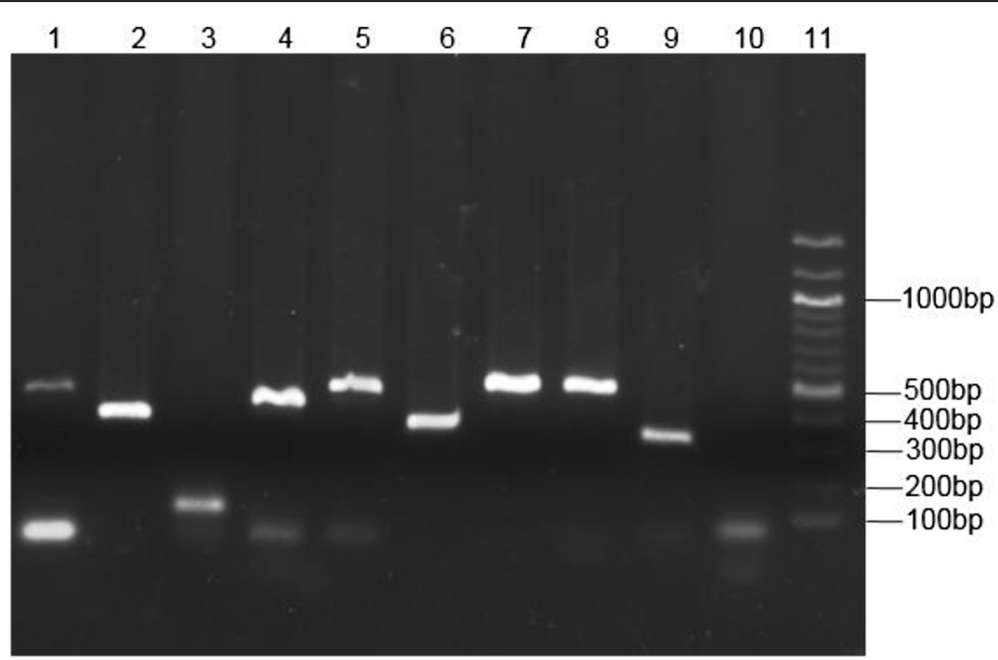

Fig. 4 Validation of novel sRNA expression by RT-PCR: Agarose gel electrophoresis of the PCR amplicon fragments of the 9 novel sRNAs. Lane 1. rev_41, Lane 2. rev_13, Lane 3. fwd_6, Lane 4. rev_11, Lane 5. fwd_72, Lane 6. fwd_44, Lane 7. fwd_42, Lane 8. rev_24, Lane 9. rev_39, Lane 10. No reverse transcriptase control, Lane 11. 100 bp DNA Ladder 


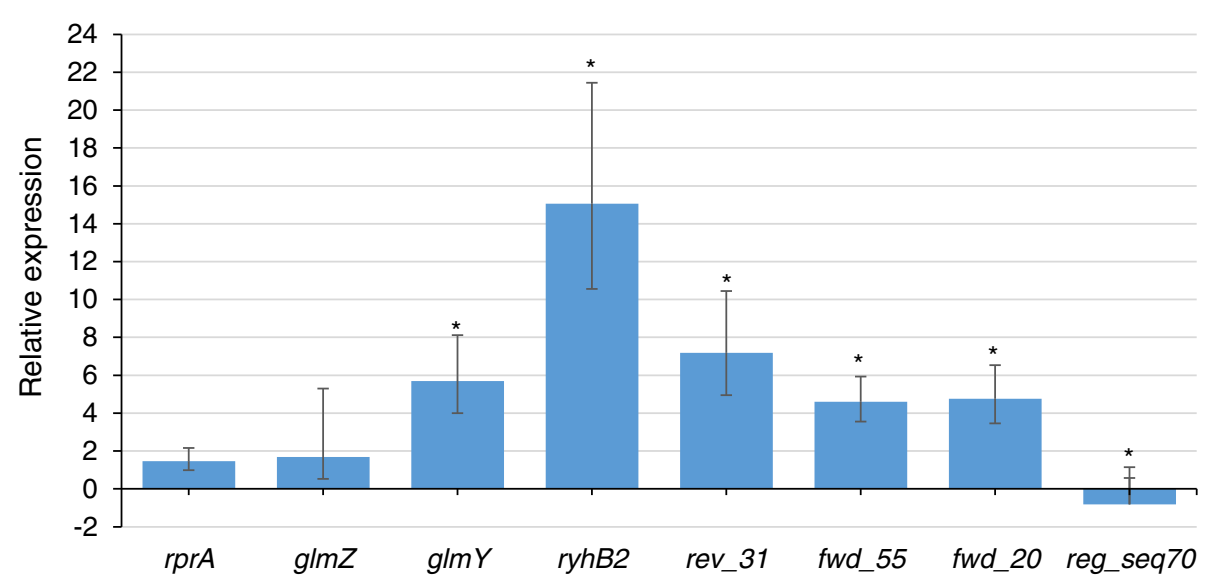

Fig. 5 RT-qPCR validation of RNA-seq expression analysis. Relative expression changes of sRNAs were determined using the 2- $\Delta \Delta \mathrm{ct}$ method by comparing expression in starvation conditions to nutrient-rich. Error bars indicate the standard error of three independent biological replicates. Asterisks represent significant difference at $p<0.05$ (Students $t$-test)

regulation by these two sRNAs modulates the transitions between carbon storage and carbon metabolism [48]. The level of $g \operatorname{lm} Y$ is increased in the absence of glucosamine-6-phosphate leading to stabilization of $g \operatorname{lm} Z$. The latter, in turn, activates $g \operatorname{lm} S$ gene expression in an anti-antisense mechanism [48]. GlmS enables cells to utilize the intermediates of glycolytic pathway including the fructose-6-phosphate for production of amino sugars. The glucosamine-6-phosphate is an essential precursor for biosynthesis of essential components of the cell envelope such as peptidoglycan and lipopolysaccharide in gram-negative bacteria. Thus, induction of $g \operatorname{lm} Y$ and $g \operatorname{lm} Z$ expression in P. atrosepticum under starvation conditions likely indicates the important role of the amino sugar metabolism in adaptive response on this bacterium.
In summary, we have shown that several sRNAs are induced under nutrient-deficient compared to nutrient-rich conditions. We have also shown that induction of these sRNA leads to induction of various genes that potentially play a role in the survival of $P$. atrosepticum. In other members of the Enterobacteriaceae family including E. coli and Salmonella, sRNAs have also been shown to play an important role in adaptation to nutrient limited condition [49]. In these bacteria, sRNAs provide a signal that triggers production of extracellular polysaccharides (EPS) which in turn are involved in biofilm formation [50]. Although $P$. atrosepticum does not readily form biofilms in vitro, the overexpression of a diguanylate cyclase $\left(\mathrm{PleD}^{*}\right)$, induced formation of biofilms suggesting that biofilm formation in this pathogen is cryptic and can be activated under optimum conditions [51]. Part of the

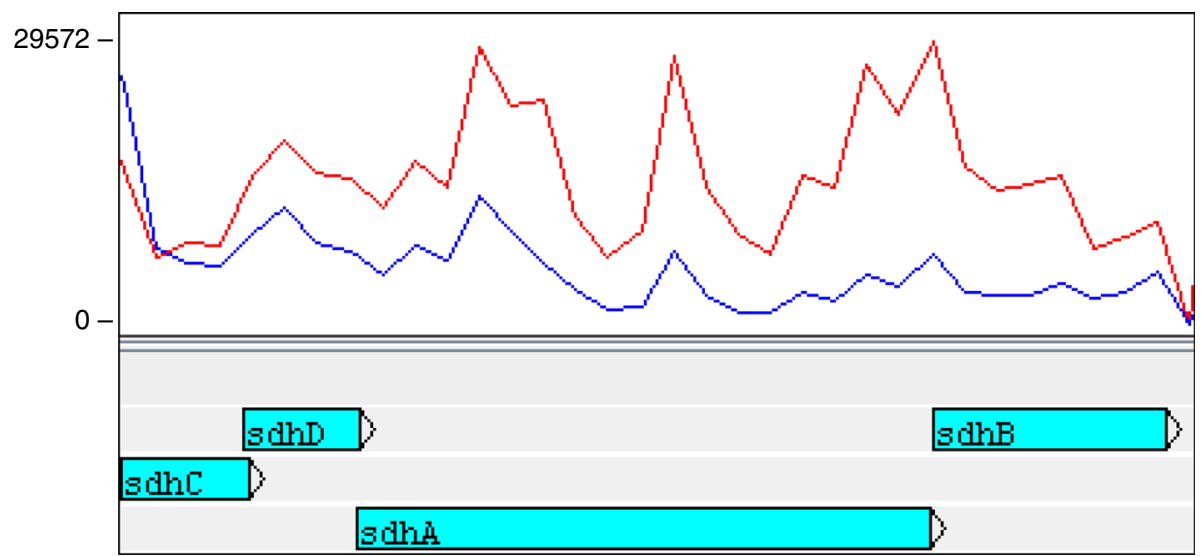

Fig. 6 The expression of the $s d h C D A B$ operon is relatively lower under starvation compared to growth promoting conditions. Reads mapped from the nutrient-rich condition are represented by the red line. The blue line represents mapped reads from the starvation conditions. Annotated features are labelled below the plot in blue blocks. The $y$-axis shows the read coverage per coding region (CDS) 
pathogenesis of $P$. atrosepticum is in xylem tissue (when causing black leg disease of potato stems). The xylem is typified by limited nutrients and as such a harsh environment that requires well defined methods of survival. Hence, it is not surprising that many xylem dwelling phytopathogens such as Xanthomonas, Clavibacter, Ralstonia and Xylella form biofilms in xylem tissues of their respective hosts. Thus, it is possible that sRNA are extensively involved in the adaptation of $P$. atrosepticum and survival in stem vasculature. Identification of this suite of sRNA will allow us to study the role that these play in survival of this phytopathogen during stem colonisation.

\section{Conclusions}

In conclusion, in this study we have used a combination of strand-specific RNA-sequencing and in silico approaches to detect and analyse sRNAs in $P$. atrosepticum SCRI1043. We demonstrated the efficiency of ssRNAseq in detecting sRNAs and determining the sRNA expression levels in response to specific bacterial growth conditions. A total of 137 sRNAs and sRNA candidates were experimentally detected in this study. We successfully determined sRNAs (that are riboswitches, transencoded sRNAs, 3' UTR sRNAs and asRNAs) that may play key roles in regulating stress responses. Most of the identified sRNAs in $P$. atrosepticum are conserved within the soft rot enterobacteria (SRE) species suggesting their importance in physiological responses for the SRE species. To our knowledge, this study constitutes the first genome/ transcriptome-wide analysis aimed at the discovery of sRNAs responsive to nutrient-deficiency (starvation) in bacteria. A significant fraction of the unravelled sRNAs appeared to be starvation responsive indicative of their importance in adaptation of bacteria to stress conditions. Determining the biological roles of these sRNAs will broaden our understanding of the diverse regulatory mechanisms they provide in modulating gene expression in $P$. atrosepticum and other SRE species during adaptation to changing environments.

\section{Methods}

Strains, culture conditions and strand-specific RNA-seq Bacterial strains, media and culture conditions

A strain of P. atrosepticum SCRI1043 [10], was used in this study. sRNA profile was analysed in bacterial cells existing under growth-promoting and starvation conditions. The cultures with inoculation titer of $2-3 \times$ $10^{6} \mathrm{CFU}$ (colony forming units) per $\mathrm{ml}$ were grown in Luria-Bertani medium [52], with aeration (200 r.p.m.) at $28{ }^{\circ} \mathrm{C}$ for $16 \mathrm{~h}$ (growth-promoting conditions). Aliquots of these cultures were used for total RNA extraction. The remaining cells were transferred (after double wash) to carbon and phosphorus deficient $\mathrm{AB}$ medium containing
$1 \mathrm{~g} \mathrm{l}^{-1} \mathrm{NH}_{4} \mathrm{Cl} ; 0.62 \mathrm{~g} \mathrm{l}^{-1} \mathrm{MgSO} 4 \times 7 \mathrm{H}_{2} \mathrm{O} ; 0.15 \mathrm{~g} \mathrm{l}^{-1} \mathrm{KCl}$; $0.013 \mathrm{~g} \mathrm{l}^{-1} \mathrm{CaCl}_{2} \times 2 \mathrm{H}_{2} \mathrm{O}$ and $0.005 \mathrm{~g} \mathrm{l}^{-1} \mathrm{FeSO}_{4} \times 7 \mathrm{H}_{2} \mathrm{O}$, $\mathrm{pH} 7.5$ and incubated under starvation conditions with initial cell density of $5.4 \times 10^{8} \pm 6.1 \times 10^{7}$ CFU per $\mathrm{ml}$ in glass vials without aeration at $28{ }^{\circ} \mathrm{C}$ [53]. Total RNA was extracted from $24 \mathrm{~h}$ starving cells.

\section{Total RNA preparation}

Total RNA was isolated from bacterial cells using the RNeasy Protect Bacteria Mini Kit (Qiagen, USA), according to the manufacturer's instructions. Contaminating DNA was removed from the samples by DNAse (Qiagen) treatment. RNA was quantified using a Qubit fluorometer (Invitrogen, USA).

\section{cDNA library construction and bacteria strand-specific RNA sequencing}

Library construction and strand-specific sequencing were carried out at the Beijing Genomics Institute (BGIShenzhen, China; http://www.genomics.cn/en/index), following the manufacturer's protocols. Briefly, the rRNA was depleted from 1 microgram of total RNA using the Ribo-Zero Magnetic Gold Kit (Epicenter). TruSeq RNA Sample Prep Kit v2 (Illumina) was used for library construction. RNA was fragmented into small pieces using Elute Prime Fragment Mix. First-strand cDNA was synthesized with First Strand Master Mix and Super Script II (Invitrogen) reverse transcription $\left(25{ }^{\circ} \mathrm{C}\right.$ for $10 \mathrm{~min} ; 42{ }^{\circ} \mathrm{C}$ for $50 \mathrm{~min} ; 70{ }^{\circ} \mathrm{C}$ for $15 \mathrm{~min}$ ). After product purification (Agencourt RNAClean XP Beads, AGENCOURT) the second-strand cDNA library was synthesized using Second Strand Master Mix and dATP, dGTP, dCTP, dUTP mix $\left(1 \mathrm{~h}\right.$ at $\left.16{ }^{\circ} \mathrm{C}\right)$. Purified fragmented cDNA was end repaired $\left(30 \mathrm{~min}\right.$ at $\left.30{ }^{\circ} \mathrm{C}\right)$ and purified with AMPureXP Beads (AGENCOURT). Addition of the poly (A) tail was done with A-tailing Mix (30 min at $37{ }^{\circ} \mathrm{C}$ ) prior to ligating sequencing adapters $\left(10 \mathrm{~min}\right.$ at $\left.30{ }^{\circ} \mathrm{C}\right)$. The second-strand cDNA was degraded using the Uracil-N-Glycosylase (UNG) enzyme $\left(10 \mathrm{~min}\right.$ at $\left.37^{\circ} \mathrm{C}\right)$ and the product purified by AMPureXP Beads (AGENCOURT). Several rounds of PCR amplification with PCR Primer Cocktail were performed to enrich the cDNA fragments and the PCR products were purified with AMPureXP Beads (AGENCOURT). Sequencing was performed using the Illumina HiSeq $^{\text {ma }} 2000$ platform with pair-end 90 base reads.

\section{Sequence read processing and experimental detection of SRNAs}

Prior to analyzing the sequencing reads, adaptors were removed and the Illumina pair-end reads were quality checked using FASTQC: Read QC and trimmed using Trim sequences (version 1.0.0) implemented within the Galaxy software [54-56]. Quality trimmed reads were 
mapped to the $P$. atrosepticum SCRI1043 genome (http://www.ncbi.nlm.nih.gov/nuccore/50118965?report= fasta) using Bowtie2 [57]. The mapped reads in SAM format were converted to sorted and indexed BAM files using SAMtools version 0.1.18 [19]. Each BAM file was split into two separate forward and reverse strand alignments using SAMtools to obtain transcriptional direction. For visualization of the data in a strand-specific manner, the genome browser Artemis [58], was used. The strand-specific RNA Sequencing data from this study have been deposited in NCBI's Gene Expression Omnibus (GEO) with the accession number GSE68547.

\section{RT-PCR validation of novel SRNA candidates}

For RT-PCR, first-strand cDNA was synthesized from $1 \mu \mathrm{g}$ of total RNA using Superscript ${ }^{\mathrm{tm}}$ III First-Strand cDNA Synthesis SuperMix kit according to the manufacturer's protocol (Invitrogen, USA). The first-strand cDNA samples were used for RT-PCR, which was performed on Bio-RAD T100 ${ }^{\mathrm{TM}}$ Thermal Cycler conventional PCR (Bio-RAD, USA). To check for genomic DNA contamination, a non reverse-transcriptase control was included. The sRNA primers were designed online using Primer3 (Additional file 8: Table S8). PCR was performed in a $25 \mu \mathrm{l}$ reaction mix containing $1 \mu \mathrm{l}$ of template cDNA ( $\sim 40 \mathrm{ng})$, Taq DNA Polymerase, $10 \times$ Taq Buffer (New England Biolabs, UK), $2.5 \mathrm{mM}$ dNTPs each and $0.5 \mu \mathrm{M}$ of forward and reverse primer each. Thermal cycling conditions were: $95{ }^{\circ} \mathrm{C}$ for $2 \mathrm{~min} ; 30$ cycles of $95{ }^{\circ} \mathrm{C}$ for $30 \mathrm{sec}, 57{ }^{\circ} \mathrm{C}$ for $30 \mathrm{sec}, 72{ }^{\circ} \mathrm{C}$ for $60 \mathrm{sec}$, and the final extension at $72{ }^{\circ} \mathrm{C}$ for $5 \mathrm{~min}$. The PCR products were analysed on $1.5 \%$ agarose gel including 100 bp DNA molecular weight ladder (NEB, UK).

\section{Differential expression analysis of sRNAs}

Artemis genome browser was used to create features of the discovered sRNAs on the $P$. atrosepticum reference genome and to make read counts for reads aligning to each strand under each growth condition. The read counts were used as input for the sRNA differential expression analysis using edgeR [39]. sRNA transcripts were considered differentially expressed provided that the p-value was $<0.05$ and q-value $<0.1$.

\section{RT-qPCR validation of RNA-seq data}

First strand cDNA synthesis was performed individually from total RNA samples from each of three biological replicates per condition using Superscript III FirstStrand cDNA Synthesis SuperMix kit (Invitrogen, USA). For RT-qPCR, $2 \mu \mathrm{l}$ of sample was added to $8 \mu \mathrm{l}$ of Applied Biosystems SYBR Green Master Mix including each primer at a concentration of $0.4 \mu \mathrm{M}$ and the reaction performed in the QuantStudio $12 \mathrm{~K}$ Flex Real-Time PCR system (Life Technologies, USA). The following cycling conditions were used: an initial denaturation at $50{ }^{\circ} \mathrm{C}$ for $5 \mathrm{~min}$ and $95{ }^{\circ} \mathrm{C}$ for $2 \mathrm{~min}$, followed by 45 cycles of $95{ }^{\circ} \mathrm{C}$ for $15 \mathrm{~s}$ and $60^{\circ} \mathrm{C}$ for $1 \mathrm{~min}$. Each sample was run in triplicate. Relative expression was measured using the comparative 2- $\Delta \Delta^{\mathrm{ct}}$ method [59] after normalizing the samples to $r e c A$ as the reference gene. Primers were designed using Primer3Plus (http://primer3plus.com/cgi-b in/dev/primer3plus.cgi) (Additional file 9: Table S9).

\section{Computational (in silico) sRNA prediction Soft rot bacteria genome sequences}

The genome sequences of six soft rot bacteria species (Pectobacterium atrosepticum SCRI1043, P. carotovorum subsp. carotovorum PC1, P. wasabiae WPP163, Pectobacterium sp. SCC3193, D. dadantii Ech703, and Dickeya zeae Ech1591 were obtained from the European Nucleotide Archive (http://www.ebi.ac.uk/ena/).

\section{Identification of RITS}

The WebGeSTer DB [60], database was used in this study to predict Rho-independent terminators (RITS) in $P$. atrosepticum SCRI1043 using default parameters. Briefly, no more than three mismatches were permitted within the stem structure and only RIT candidates with the highest $\Delta \mathrm{G}$ score $(\Delta \mathrm{G}<=-12.0 \mathrm{kcal} / \mathrm{mol})$ were considered. Coordinates for putative RITs were obtained from the WebGeSTer DB, and java scripts were used to extract the sequences $200 \mathrm{nt}$ upstream of the terminators (including the stem loop and tail sequences of the terminator). These sequences were considered as putative sRNA candidates and used in downstream sRNA prediction analysis. Additionally, known sRNAs within the $P$. atrosepticum SCRI1043 genome sequence were searched in the SIPHT web interface (published annotations) [28].

\section{sRNA conservation analysis}

The conservation of sRNA sequences detected using deep sequencing was determined by similarity analysis against sequences of complete genomes of five soft rot Enterobacteriaceae species using YASS [29], a sequence similarity search tool, with standard parameters.

\section{Classification of sRNA}

Following the model implemented for Escherichia coli [27], custom scripts written in java were used to classify the predicted sRNA candidates into five non-coding RNA groups based on their position in relation to adjacent CDSs. Briefly, the first nucleotide in each RIT was used as the representative position of each sRNA candidate. To determine asRNA, the reference nucleotide on the opposite DNA strand had to be at least +15 nt relative to the ATG codon to $-50 \mathrm{nt}$ with respect to the stop codon. For 5' UTR, sRNA candidates had to be on 
the same DNA strand as the CDS and in a distance of $<-100$ nt upstream the ATG codon and for 3' UTR between +60 and +200 nt downstream of the stop codon. The rest of the remaining putative sRNAs were considered as IGR candidates if they were outside a CDS.

\section{Additional files}

Additional file 1: Table S1. Complete list of RNA-seq detected SRNAs (XLSX $19 \mathrm{~kb}$ )

Additional file 2: Table S2. Predicted transcription start sites of RNA-seq detected sRNAs (XLSX $9114 \mathrm{~kb})$

Additional file 3: Table S3. List of in silico predicted SRNAs using RITs from WebGester DB. S2A: Forward strand predictions. S2B: Complementary strand predictions (XLSX $30 \mathrm{~kb}$ )

Additional file 4: Table S4. Combined list of predicted sRNA using SIPHT and RITs from WebGester DB. S3A: Matches of in silico predictions with SIPHT (forward strand) S3B: Matches of in silico predictions with SIPHT (complementary strand) (XLSX $15 \mathrm{~kb}$ )

Additional file 5: Table S5. Conservation analysis in Soft Rot Enterobacteriaceae (XLSX $16 \mathrm{~kb}$ )

Additional file 6: Table S6. Confirmation of RT-PCR amplicons by sequencing and BLASTn against respective sRNA sequences (XLSX $9 \mathrm{~kb}$ )

Additional file 7: Table S7. Differentially expressed sRNA under nutrient-rich and starvation conditions (XLSX $19 \mathrm{~kb}$ )

Additional file 8: Table S8. List of primers used for RT-PCR validation of novel sRNAs (DOCX $12 \mathrm{~kb}$ )

Additional file 9: Table S9. List of primers used for RT-qPCR validation of RNA-seq expression data (DOCX $12 \mathrm{~kb}$ )

\section{Abbreviations}

3' UTR: 3' untranslated regions; 5' UTR: 5' untranslated regions; asRNAs: antisense RNAs; BLAST: basic local alignment search tool; CDSs: protein-coding regions; CFU: colony forming units; EPS: extracellular polysaccharides; IGR: intergenic regions; mRNA: messenger RNA; ORF: open reading frame; RITs: rho-independent terminators; RPKM: reads per kilobase of transcript per Million mapped reads; SRE: soft rot enterobacteriaceae; SRNA: small RNA; ssRNA-seq: strand-specific RNA sequencing; TPP: thiamine pyrophosphate; TSS: transcriptional start sites.

\section{Competing interests}

The authors declare that they have no competing interests.

\section{Authors' contributions}

SK, VG and LNM conceived the study. SK carried out bioinformatics data analyses. AMR and SN experimental validation of novel sRNAs. SK and AMR performed GPCR analysis. ER advised on bioinformatics approaches. PRJB critically reviewed the manuscript. All authors wrote the manuscript. All authors read and approved the final manuscript.

\section{Acknowledgements}

The authors would like to acknowledge funding from The National Research Foundation (NRF) Thuthuka Grant UID: 69362; UID BFG 93685 and NRF South Africa Russia Bilateral Grant UID: 75252 and from the Russian Foundation for Basic Research (Research Project No. 15-04-02380). The University of Pretoria is acknowledged for providing a studentship for S. Kwenda. V. Gorshkov is supported by The Russian Science Foundation (Project No. 15-14-10022).

\section{Author details}

'Department of Microbiology and Plant Pathology, Forestry and Agricultural Biotechnology Institute (FABI), University of Pretoria, Pretoria, South Africa. ${ }^{2}$ Kazan Institute of Biochemistry and Biophysics, Kazan Scientific Center, Russian Academy of Sciences, Kazan, Russia. ${ }^{3}$ Department of Botany and Plant Physiology, Kazan Federal University, Kazan, Russia. ${ }^{4}$ Department of Genetics, Forestry and Agricultural Biotechnology (FABI), University of
Pretoria, Pretoria, South Africa. ${ }^{5}$ Genomics Research Institute, Centre for Microbial Ecology and Genomics (CMEG), University of Pretoria, Pretoria, South Africa. ${ }^{6}$ Division of Plant Sciences, College of Life Sciences, University of Dundee (at The James Hutton Institute), Errol Road, Invergowrie, Dundee DD25DAScotland, UK.

Received: 3 September 2015 Accepted: 6 January 2016

Published online: 12 January 2016

\section{References}

1. Waters LS, Storz G. Regulatory RNAs in bacteria. Cell. 2009;136(4):615-28.

2. Wilms I, Overlöper A, Nowrousian M, Sharma CM, Narberhaus F. Deep sequencing uncovers numerous small RNAs on all four replicons of the plant pathogen Agrobacterium tumefaciens. RNA Biol. 2012;9(4):446

3. Peer $\mathrm{A}$, Margalit H. Accessibility and evolutionary conservation mark bacterial small-RNA target-binding regions. J Bacteriol. 2011;193(7):1690-701.

4. Gottesman S, Storz G. Bacterial small RNA regulators: versatile roles and rapidly evolving variations. Cold Spring Harb Perspect Biol. 2011;3(12):a003798.

5. Fozo EM, Hemm MR, Storz G. Small toxic proteins and the antisense RNAs that repress them. Microbiol Mol Biol Rev. 2008;72(4):579-89.

6. Croucher NJ, Fookes MC, Perkins TT, Turner DJ, Marguerat SB, Keane T, et al. A simple method for directional transcriptome sequencing using Illumina technology. Nucleic Acids Res. 2009;37(22):e148.

7. Graveley BR. Molecular biology: power sequencing. Nature. 2008:453(7199): $1197-8$

8. Soutourina OA, Monot M, Boudry P, Saujet L, Pichon C, Sismeiro O, et al. Genome-Wide Identification of Regulatory RNAs in the Human Pathogen Clostridium difficile. PLoS Genet. 2013;9(5), e1003493.

9. Cho SH, Lei R, Henninger TD, Contreras LM. Discovery of ethanol responsive small RNAs in Zymomonas mobilis. Appl Environ Microbiol. 2014;80(14): 4189-98. doi:10.1128/AEM.00429-14.

10. Bell K, Sebaihia M, Pritchard L, Holden M, Hyman L, Holeva M, et al. Genome sequence of the enterobacterial phytopathogen Erwinia carotovora subsp. atroseptica and characterization of virulence factors. Proc Natl Acad Sci U S A. 2004:101(30):11105-10.

11. Charkowski A, Blanco C, Condemine G, Expert D, Franza T, Hayes C, et al. The role of secretion systems and small molecules in soft-rot enterobacteriaceae pathogenicity. Annu Rev Phytopathol. 2012;50:425-49.

12. Gorshkov V, Petrova O, Gogoleva N, Gogolev Y. Cell-to-cell communication in the populations of enterobacterium Erwinia carotovora ssp. atroseptica SCRI1043 during adaptation to stress conditions. FEMS Immunol Med Microbiol. 2010;59(3):378-85

13. Gorshkov WY, Petrova O, Mukhametshina N, Ageeva M, Mulyukin A, Gogolev YV. Formation of "nonculturable" dormant forms of the phytopathogenic enterobacterium Erwinia carotovora. Microbiology. 2009;78(5):585-92.

14. Petrova O, Gorshkov V, Daminova A, Ageeva M, Moleleki L, Gogolev Y. Stress response in Pectobacterium atrosepticum SCRI 1043 under starvation conditions: adaptive reactions at a low population density. Res Microbiol. 2013;165(2):119-27.

15. Cui Y, Chatterjee A, Chatterjee AK. Effects of the two-component system comprising GacA and GacS of Erwinia carotovora subsp. carotovora on the production of global regulatory rsmB RNA, extracellular enzymes, and harpinEcc. Mol Plant-Microbe Interact. 2001;14(4):516-26.

16. Liu Y, Cui Y, Mukherjee A, Chatterjee AK. Characterization of a novel RNA regulator of Erwinia carotovora ssp. carotovora that controls production of extracellular enzymes and secondary metabolites. Mol Microbiol. 1998; 29(1):219-34.

17. Mukherjee A, Cui Y, Liu Y, Dumenyo CK, Chatterjee AK. Global regulation in Erwinia species by Erwinia carotovora rsmA, a homologue of Escherichia coli csrA: repression of secondary metabolites, pathogenicity and hypersensitive reaction. Microbiology. 1996;142(2):427-34.

18. Gogoleva N, Shlykova L, Gorshkov VY, Daminova A, Gogolev YV. Effect of topology of quorum sensing-related genes in Pectobacterium atrosepticum on their expression. Mol Biol. 2014;48(4):583-9.

19. Li H, Handsaker B, Wysoker A, Fennell T, Ruan J, Homer N, et al. The sequence alignment/map format and SAMtools. Bioinformatics. 2009; 25(16):2078-9.

20. Perkins $T$ T, Kingsley RA, Fookes MC, Gardner PP, James KD, Yu L, et al. A strand-specific RNA-Seq analysis of the transcriptome of the typhoid bacillus salmonella typhi. PLoS Genet. 2009;5(7), e1000569. 
21. Guo MS, Updegrove TB, Gogol EB, Shabalina SA, Gross CA, Storz G. MicL, a new $\sigma E$-dependent sRNA, combats envelope stress by repressing synthesis of Lpp, the major outer membrane lipoprotein. Genes Dev. 2014;28(14):1620.

22. Chao Y, Papenfort K, Reinhardt R, Sharma CM, Vogel J. An atlas of Hfq-bound transcripts reveals $3^{\prime}$ UTRs as a genomic reservoir of regulatory small RNAs. EMBO J. 2012;31(20):4005-19.

23. Miyakoshi M, Chao Y, Vogel J. Regulatory small RNAs from the $3^{\prime}$ regions of bacterial mRNAs. Curr Opin Microbiol. 2015;24:132-9.

24. Nawrocki EP, Burge SW, Bateman A, Daub J, Eberhardt RY, Eddy SR, et al. Rfam 12.0: updates to the RNA families database. Nucleic Acids Res. 2014;43: D130-7. doi:10.1093/nar/gku1063.

25. Kulkarni RV, Kulkarni PR. Computational approaches for the discovery of bacterial small RNAs. Methods. 2007;43(2):131-9.

26. Livny J, Waldor MK. Identification of small RNAs in diverse bacterial species. Curr Opin Microbiol. 2007;10(2):96-101.

27. Pichon C, Du Merle L, Caliot ME, Trieu-Cuot P, Le Bouguénec C. An in silico model for identification of small RNAs in whole bacterial genomes: characterization of antisense RNAs in pathogenic Escherichia coli and Streptococcus agalactiae strains. Nucleic Acids Res. 2012;40(7):2846-61.

28. Livny J. Bioinformatic Discovery of Bacterial Regulatory RNAs Using SIPHT. In: Bacterial Regulatory RNA. Springer. 2012. p. 3-14.

29. Noé L, Kucherov G. YASS: enhancing the sensitivity of DNA similarity search. Nucleic Acids Res. 2005;33 suppl 2:W540-3.

30. Vockenhuber M-P, Sharma CM, Statt MG, Schmidt D, Xu Z, Dietrich S, et al. Deep sequencing-based identification of small non-coding RNAs in Streptomyces coelicolor. RNA Biol. 2011:8(3):468.

31. Burge SW, Daub J, Eberhardt R, Tate J, Barquist L, Nawrocki EP, et al. Rfam 11.0: 10 years of RNA families. Nucleic Acids Res. 2013:41(D1):D226-32.

32. Cros M-J, De Monte A, Mariette J, Bardou P, Grenier-Boley B, Gautheret D, et al. RNAspace. org: An integrated environment for the prediction, annotation, and analysis of ncRNA. RNA. 2011;17(11):1947-56.

33. Hofacker IL, Fontana W, Stadler PF, Bonhoeffer LS, Tacker M, Schuster P. Fast folding and comparison of RNA secondary structures. Monatshefte für Chemie/Chemical Monthly. 1994;125(2):167-88.

34. Nudler $\mathrm{E}$, Mironov AS. The riboswitch control of bacterial metabolism. Trends Biochem Sci. 2004;29(1):11-7.

35. Miranda-Ríos J, Navarro M, Soberón M. A conserved RNA structure (thi box) is involved in regulation of thiamin biosynthetic gene expression in bacteria. Proc Natl Acad Sci. 2001;98(17):9736-41.

36. Chen Y, Indurthi DC, Jones SW, Papoutsakis ET. Small RNAs in the genus Clostridium. MBio. 2011;2(1):e00340-00310.

37. Arnvig KB, Comas I, Thomson NR, Houghton J, Boshoff HI, Croucher NJ, et al. Sequence-based analysis uncovers an abundance of non-coding RNA in the total transcriptome of Mycobacterium tuberculosis. PLoS Pathog. 2011; 7(11), e1002342.

38. Arnvig K, Young D. Non-coding RNA and its potential role in Mycobacterium tuberculosis pathogenesis. RNA Biol. 2012;9(4):427-36.

39. Robinson MD, McCarthy DJ, Smyth GK. edgeR: a Bioconductor package for differential expression analysis of digital gene expression data. Bioinformatics. 2010;26(1):139-40.

40. Feng J, Meyer CA, Wang Q, Liu JS, Liu XS, Zhang Y. GFOLD: a generalized fold change for ranking differentially expressed genes from RNA-seq data. Bioinformatics. 2012;28(21):2782-8.

41. Repoila F, Majdalani N, Gottesman S. Small non-coding RNAs, co-ordinators of adaptation processes in Escherichia coli: the RpoS paradigm. Mol Microbiol. 2003;48(4):855-61.

42. Majdalani N, Chen S, Murrow J, St John K, Gottesman S. Regulation of RpoS by a novel small RNA: the characterization of RprA. Mol Microbiol. 2001; 39(5):1382-94

43. Hengge-Aronis R. Signal transduction and regulatory mechanisms involved in control of the oS (RpoS) subunit of RNA polymerase. Microbiol Mol Biol Rev. 2002;66(3):373-95

44. Mandin P, Gottesman S. Integrating anaerobic/aerobic sensing and the general stress response through the ArcZ small RNA. EMBO J. 2010;29(18): 3094-107.

45. Richards GR, Vanderpool CK. Molecular call and response: the physiology of bacterial small RNAs. Biochimica et Biophysica Acta (BBA)-Gene Regulatory Mechanisms. 2011;1809(10):525-31.

46. Hoe C-H, Raabe CA, Rozhdestvensky TS, Tang T-H. Bacterial sRNAs: regulation in stress. Int J Med Microbiol. 2013;303(5):217-29.
47. Urban JH, Vogel J. Two seemingly homologous noncoding RNAs act hierarchically to activate glmS mRNA translation. PLoS Biol. 2008;6(3), e64.

48. Bobrovskyy M, Vanderpool CK. Regulation of bacterial metabolism by small RNAs using diverse mechanisms. Annu Rev Genet. 2013;47:209-32.

49. Mika F, Hengge R. Small regulatory RNAs in the control of motility and biofilm formation in E. coli and Salmonella. Int J Mol Sci. 2013:14(3):4560-79.

50. Mika F, Hengge R. Small RNAs in the control of RpoS, CsgD, and biofilm architecture of Escherichia coli. RNA Biol. 2014;11(5):494-507.

51. Pérez-Mendoza D, Coulthurst SJ, Sanjuán J, Salmond GP. NAcetylglucosamine-dependent biofilm formation in Pectobacterium atrosepticum is cryptic and activated by elevated c-di-GMP levels. Microbiology. 2011;157(12):3340-8.

52. Sambrook J, Fritsch EF, Maniatis T. Molecular cloning, vol. 2. New York: Cold Spring Harbor Laboratory Press; 1989.

53. Petrova O, Gorshkov V, Daminova A, Ageeva M, Moleleki LN, Gogolev Y. Stress response inPectobacterium atrosepticumSCRI1043 under starvation conditions: adaptive reactions at a low population density. Res Microbiol. 2014;165(2):119-27.

54. Blankenberg D, Kuster GV, Coraor N, Ananda G, Lazarus R, Mangan M, et al. Galaxy: a web-based genome analysis tool for experimentalists. Curr Protoc Mol Biol. 2010;19(10):11-19. 10. 21.

55. Giardine B, Riemer C, Hardison RC, Burhans R, Elnitski L, Shah P, et al. Galaxy: a platform for interactive large-scale genome analysis. Genome Res. 2005; 15(10):1451-5.

56. Goecks J, Nekrutenko A, Taylor J. Galaxy: a comprehensive approach for supporting accessible, reproducible, and transparent computational research in the life sciences. Genome Biol. 2010;11(8):R86.

57. Langmead B, Salzberg SL. Fast gapped-read alignment with Bowtie 2. Nat Methods. 2012;9(4):357-9.

58. Rutherford K, Parkhill J, Crook J, Horsnell T, Rice P, Rajandream M-A, et al. Artemis: sequence visualization and annotation. Bioinformatics. 2000;16(10): 944-5.

59. Livak KJ, Schmittgen TD. Analysis of relative gene expression data using real-time quantitative PCR and the $2-\Delta \Delta C T$ method. Methods. 2001;25(4): 402-8.

60. Mitra A, Kesarwani AK, Pal D, Nagaraja V. WebGeSTer DB-a transcription terminator database. Nucleic Acids Res. 2011:39 suppl 1:D129-35.

\section{Submit your next manuscript to BioMed Central and we will help you at every step:}

- We accept pre-submission inquiries

- Our selector tool helps you to find the most relevant journal

- We provide round the clock customer support

- Convenient online submission

- Thorough peer review

- Inclusion in PubMed and all major indexing services

- Maximum visibility for your research

Submit your manuscript at www.biomedcentral.com/submit
) Biomed Central 